\title{
Criando uma plataforma para Projetos de Aprendizagem: desafios e reflexões no desenvolvimento do AMADIS ${ }^{1}$
}

\author{
Juliano de Vargas Bittencourt ${ }^{1}$, James Zortéa, Lilian Junqueira ${ }^{1}$, \\ Robson Mendonça' ${ }^{1}$, Marcus Vinícius Basso', \\ Léa da Cruz Fagundes ${ }^{1}$, Crediné Silva de Menezes ${ }^{2}$, Rosane Aragon de Nevado ${ }^{1}$ \\ ${ }^{1}$ Laboratório de Estudos Cognitivos - LEC/UFRGS \\ ${ }^{2}$ Departamento de Informática - UFES \\ juliano@lec.ufrgs.br, james@lec.ufrgs.br, lilian@lec.ufrgs.br, robson@lec.ufrgs.br, \\ mbasso@ufrgs.br, leafaung@ufrgs.br, credine@inf.ufes.br, rosane@edu.ufrgs.br
}

\section{Resumo}

A plataforma virtual de aprendizagem AMADIS tem como objetivo suportar o trabalho por uma pedagogia de "Projetos de Aprendizagem" de forma presencial e a distância. Desta forma, ela afasta-se de um modelo de plataformas centradas na realização de cursos e na publicação de materiais. A implementação de uma ferramenta para projetos tem criado inúmeros novos problemas de design que não se restringem à questão de usabilidade, mas se relacionam principalmente com a coerência do sistema com sua proposta pedagógica e epistemológica. Este artigo relata alguns dos desafios que a equipe do LEC/UFRGS vem enfrentando na pesquisa relacionada ao desenvolvimento da plataforma, bem como algumas das soluções encontradas e das reflexões geradas.

Palavras-chaves: ambientes virtuais de aprendizagem, educação a distância, desenvolvimento de projetos de aprendizagem, ferramentas de autoria.

\section{Resúmen}

La Plataforma virtual de aprendizaje AMADIS tiene por objetivo soportar el trabajo por una pedagogía de "Proyecto de Aprendizaje" de forma presencial y a distancia. Así, ella se distancia de un modelo de plataformas centradas en la realización de cursos y en la publicación de materiales. La implementación de una herramienta para proyectos ha creado incontables nuevos problemas de diseño que no se limitan a la cuestión de usabilidad, pero se relacionan principalmente con la coerencia del sistema con su propuesta pedagógica y epistemológica. Este artigo relata algunos de los desafíos que el equipo del LEC/UFRGS ha enfrentado en la pesquisa relacionada con el desarrollo de la plataforma, así como algunas de las soluciones encontradas y de las reflexiones generadas.

Palabras clave: ambientes virtuales de aprendizaje, educación a distancia, desarrollo de proyectos de aprendizaje, herramientas de autoría

\section{Introdução}

A discussão sobre o uso de ferramentas telemáticas para auxiliar as práticas

\footnotetext{
1 Os resultados das pesquisas aqui relatadas foram financiadas com recursos do Fundo Setorial de Informática - CTInfo, por meio do edital de pesquisa e desenvolvimento com inovação tecnológica em Software Livre. V. $4 \mathrm{~N}^{\mathrm{o}} 1$, Julho, 2006
} 
pedagógicas vem sendo recorrentes na área da Educação. Cabe lembrar que, apesar disso, muitas vezes o debate centra-se na "inovação tecnológica", abandonando-se a análise sobre a proposta pedagógica que dá suporte a esse novo instrumento. O que pode ser observado, então, é a repetição de um modelo de educação tradicional, centrado na heteronomia do professor, mas com um invólucro tecnológico interativo.

Antes de se fazer uma transposição direta das práticas pedagógicas correntes para o contexto da Internet, é preciso valorizar as especificidades de cada meio. Somente a partir do reconhecimento das características do meio digital, é possível aproveitar ao máximo as ferramentas disponíveis para instigar o aluno a construir o seu conhecimento.

Além disso, o uso contextualizado da Internet, visando valorizar a cooperação entre os aprendizes e aliar as práticas pedagógicas que instiguem o aluno a desenvolver sua autonomia, possibilita uma riqueza de interação que acaba por motivá-lo a socializar suas construções epistêmicas.

O desenvolvimento do conhecimento resulta em atividade operatória do sujeito, que o constrói quando está em interação com o meio, com os outros sujeitos e com os objetos de conhecimento de que ele deseje apropriar-se (Fagundes et al,1999). No entanto, para que se efetive a construção de conhecimento, não basta apenas agir sobre o meio, é necessário, tomar consciência dessa ação. "Cooperação pressupõe descentração do pensamento e coordenação de diferentes pontos de vista”. (Fagundes, 2005)

A proposta da plataforma AMADIS $^{2}$ (Nevado, 2001) é dar suporte à Pedagogia de Projetos de Aprendizagem, expandindo o conceito de aprendizagem ao cotidiano do sujeito. Em vista disso, não se pretende, através da plataforma, atrelar o processo de aprendizagem somente às atividades de ensino e de treinamento para o desenvolvimento de cursos em EAD. Mais do que isso, o que se pretende com o AMADIS é desenvolver a autonomia e o senso de cooperação do aprendiz, a fim de que este seja agente e coopere para a construção de conhecimento da comunidade em que ele se insere.

Nesse contexto, o ambiente permite ao usuário o exercício da autoria e da cooperação, na medida em que oferece ferramentas centradas no desenvolvimento de projetos de aprendizagem, que serão efetuados pelas pessoas que pretendem construir conhecimentos.

A equipe do LEC/UFRGS, utilizando-se das possibilidades de redes virtuais abertas para o trabalho cooperativo e, somando a isso, da possibilidade de interação do aprendiz com objetos educacionais e com pessoas, criou um espaço de troca de idéias que fomenta a produção de conhecimento concretizada em projetos. É a partir dessa perspectiva que se pretende, através do ambiente, fomentar uma mudança no paradigma educacional para uma compreensão mais aberta, conectada ao cotidiano de trocas interindividuais dos sujeitos.

Neste artigo, serão discutidas algumas das ferramentas implementadas na plataforma AMADIS, afim de que se demonstre uma possibilidade de uso da tecnologia a favor de um conceito de aprendizagem embasado na Pedagogia de Projetos. Numa primeira etapa, discorre-se sobre as mudanças efetivadas no ambiente em relação aos menus de navegação. Em seguida, relata-se sobre a construção, na plataforma, da área de Webfólio ( $c f$. Nevado, Magdalena e Costa, 1999)., um espaço pessoal do usuário, no qual ele insere e administra suas produções. Por fim, descreve-se o uso de técnicas de visualização de informações para representar o fluxo de interação dos usuários no

2 O AMADIS encontra-se na sua versão 1.2. Durante este artigo serão realizadas algumas comparações entre a versão atual e a anterior, denominada AMADIS 1.0.

$2 \longrightarrow$ V. 4 No 1, Julho, 2006 
ambiente.

\section{Investigações Realizadas sobre o AMADIS}

Como ponto de partida desta pesquisa, que aborda as principais reformulações do ambiente AMADIS, tomou-se como referência as seguintes questões:

A arquitetura de conexões do AMADIS é coerente com os conceitos pedagógicos propostos?

Como complexificar a navegação do sujeito-construtor ${ }^{3}$, ampliando a gama de possibilidades de percursos não-lineares no ambiente, mas também não tornando a interface incompreensível? ambiente?

Como facilitar a cooperação entre os usuários, incentivando a interação no

Como tornar observável ao usuário a complexidade das relações que compõem o ambiente, através de um mapeamento dinâmico?

\section{Confusão entre ferramentas e categorias}

A barra superior de Navegação do AMADIS 1.0 listava os seguintes tópicos numa mesma disposição visual: Página Inicial, Projetos, Cursos, Diário, Chat, Fórum, Comunidade, Correio e Administração. Essa orientação reunia, numa mesma hierarquia visual, o acesso a ações de naturezas distintas. Havia uma aglomeração de ferramentas de comunicação somada a tópicos de localização e de estruturação. Essa organização acarretava conflitos na navegação, pois era difícil para o usuário distinguir entre área pessoal e área coletiva do AMADIS.

Após várias reflexões e discussões da equipe de desenvolvimento, resolveu-se adotar uma nova solução para as barras navegacionais: o usuário contaria, então, no menu lateral esquerdo, com a sua área pessoal; enquanto que, na barra superior, se apresentariam os tópicos gerais de estruturação e localização do ambiente.

Através dessa reformulação no menu lateral esquerdo, foi possível aproximar o sujeito das suas produções numa área específica. Para tanto, foram disponibilizadas ferramentas como: Webfólio, Diário, Arquivos, Projetos, Amigos e Comunidades, todas elas particulares do usuário. Essas mudanças diversificaram os percursos possíveis do usuário para acessar as informações relacionadas à sua produção.

Além disso, a reformulação da barra superior evidencia as diferentes áreas gerais do ambiente: Projetos, Pessoas e Comunidades. Dessa forma, foi possível organizar o ambiente em três áreas distintas, facilitando ao usuário a sua localização. (Ver Figura $1)$.

\footnotetext{
sujeito-construtor: O participante do universo AMADIS é convidado a cooperar/construir, através da interface AMADIS, no desenvolvimento do diverso de pesquisas suportados pelo ambiente. Nesse contexto, cada funcionalidade de navegação do ambiente é criada com intenção de ampliar as possibilidades de apreender do colaborador. V. $4 \mathrm{~N}^{\mathrm{o}} 1$, Julho, 2006
} 


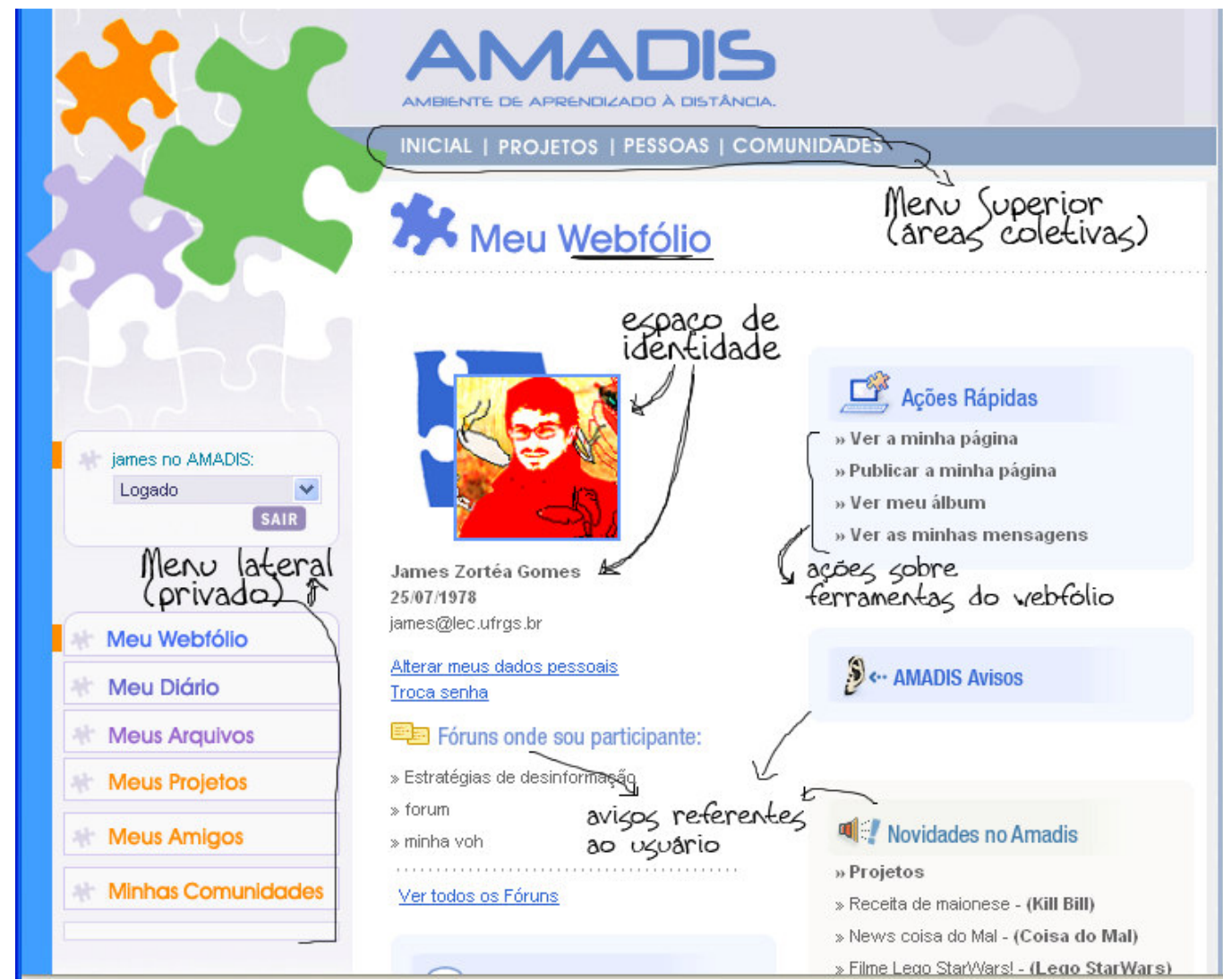

Figura 1: Observações sobre a tela do Webfólio do AMADIS 1.2

\section{O processo de re-compreensão do Webfólio como território de apropriação do indivíduo no ambiente AMADIS}

A página de Webfólio é o principal espaço de organização da produção do indivíduo dentro do AMADIS. É a partir dela que o usuário pode acessar as suas atividades, localizando e explorando os seus espaços de interação. O Webfólio, na versão AMADIS 1.2, dá acesso às diversas ações do indivíduo sobre a plataforma, mapeando os seus processos de construção. Optou-se por exibir a página de Webfólio logo após do login, pelo fato de assim se expor, ao sujeito, o reconhecimento e a organização dos seus espaços de construção e trocas.

Na versão AMADIS 1.0, o Webfólio apresentava um layout deficitário de ferramentas que proporcionassem um espaço de construção de identidade do sujeito $\mathrm{O}$ Webfólio não se explicitava como um campo de apropriação do sujeito. Não havia espaço para registrar ações que interferissem sobre a visualidade da tela, tais como: inclusão de fotos e definição de um perfil, o que possibilitaria a demarcação do sujeito como um ponto de referência do ambiente. Nesse sentido, fez-se necessário flexibilizar essa estrutura, afim de que o usuário significasse o espaço como seu, estimulando-o a se apropriar do ambiente. Foi a partir desta nova concepção que se desenvolveu o AMADIS 1.2.

A área foi reorganizada seguindo alguns pressupostos da versão anterior, como mapear ao sujeito todo seu material intelectual, porém, enfatizou-se uma nova abordagem: o Webfólio como um espaço próprio para a construção da identidade do 
sujeito.

Nesse espaço, encontram-se as ferramentas de edição dos dados pessoais, que se caracterizam pela descrição de um perfil e pela foto de representação do usuário. Foram adicionadas também "caixas de funções" que informam sobre as novidades das áreas de Projetos e de Comunidades vinculadas ao usuário. Além disso, foram inseridos alertas de mensagens direcionadas ao usuário por outros membros do ambiente. Deste modo, toda ação do usuário pode ser referenciada no seu Webfólio, por meio de um link, o que facilita o reconhecimento e a aproximação das pessoas que contribuem nas trocas de informação.

Por fim, cabe ressaltar que, nesta versão do AMADIS 1.2, são apresentadas configurações distintas de visualização para uma mesma página de Webfólio. Isto é, caso o usuário esteja visualizando o seu próprio Webfólio, este apresenta todas as ferramentas de edição e as suas mensagens particulares, enquanto que, para outro usuário que visite este Webfólio, a página apresentaria apenas a visualização das produções e os dados pessoais do autor, permitindo o envio de mensagens a ele. Nesse contexto, cada Webfólio exibe um perfil próprio dos sujeitos, que indexa não somente seus dados pessoais e fotos, mas também suas principais contribuições e vinculações com Comunidades e Projetos.

\section{Utilização de Técnicas de visualização de dados como elementos de interface}

Quando uma plataforma está organizada seguindo uma estrutura de cursos, o aprendiz tem contato apenas com as atividades que pode realizar. Desta forma, a parcela de dados que ele tem acesso é sempre limitada. Por outro lado, numa plataforma como o AMADIS, deseja-se que os sujeitos ajam de uma forma investigativa, explorando os dados disponíveis, observando as produções de outros sujeitos e interagindo com estas. A partir da ampliação das possibilidades de ação do usuário sobre a plataforma, aumenta-se a quantidade informações disponíveis para consulta e manipulação. Além disso, essa proposta também desloca as interações de locais claramente circunscritos pela hierarquia rígida das relações Curso-Disciplina-Turma, tão comuns em outras plataformas. Isso causa uma pulverização das interações dos usuários, fato que deve ser controlado de forma a não inviabilizar o trabalho de professores, orientadores e tutores.

Para enfrentar tal desafio, foram empregadas no AMADIS 1.2 diversas técnicas de Visualização de Informações ( $c f$. GERSHON, 1997) com o objetivo de dar uma maior visibilidade aos diversos tipos de informação do ambiente. Além disso, ao utilizar tais técnicas, também se deseja criar uma alternativa para os diversos sistemas de estatísticas, comuns em plataformas para EAD. Como será visto, o uso de visualização de informações é uma forma de evidenciar os aspectos qualitativos das informações, bem como as suas relações. Na presente subseção, examinaremos dois usos de tais técnicas dentro do AMADIS.

Durante o Programa ECSIC, um dos problemas observados foi a dificuldade dos usuários em observar as ações que estavam ocorrendo sobre o ambiente, em virtude do grande volume de informações disponíveis. Nesse contexto, várias formas de busca e de navegação sobre esses dados foram implementadas, visando facilitar a exploração dos dados. Porém, os usuários continuavam a apresentar dificuldades em construir uma "imagem" da totalidade do ambiente. A complexidade das relações e a intensa atividade 
ocorrida nas diversas ferramentas de interação dos projetos não eram facilmente observáveis pelos usuários/aprendizes, o que lhes deixava com uma visão fragmentada e desarticulada do ambiente. Para visualizar o que estava ocorrendo dentro daquele espaço virtual, os sujeitos precisavam visitar cada um dos diferentes projetos e seus respectivos fóruns, chats e páginas, o que lhes exigia um esforço de representação para construir mentalmente uma imagem desse "lugar". Tarefa esta de caráter ininterrupto, devido ao constante fluxo de interações que ali se desenrolavam.

Como forma de compreender esse problema, buscou-se auxilio nas investigações realizadas por Boyd et al. (2002) sobre espaços sociais virtuais. Nesses estudos, o autor aplica o conceito de legibilidade de espaços sociais, utilizado no planejamento urbano, através do desenvolvimento da interface para uma ferramenta de acesso ao usenet, denominada Loom.

Ao "tomar emprestado" da arquitetura o conceito de legibilidade e aplicá-lo na visualização de "paisagens de informações" 4 , Boyd destaca a importância da interface fornecer "pistas" e padrões que ajudem o usuário a compreender o espaço de um ambiente, a sua história e as pessoas que nele habitam. Para realizar isso, ele utiliza representações visuais com fortes laços com o mundo físico ou com a cultura dos usuários.

Os estudos de Boyd ajudaram a equipe de desenvolvimento a compreender a dificuldade enfrentada por seus usuários. A interface do AMADIS não proporcionava meios para que os usuários observassem as mudanças que ocorriam naquele ambiente. No mundo físico, quando um sujeito realiza uma inspeção visual sobre um espaço do qual ele se manteve afastado durante algum tempo, possivelmente ele conseguirá apontar algumas das mudanças que ocorreram. No entanto, em um espaço virtual, cabe ao projetista da interface criar meios para que se torne possível a visualização dessas mudanças. Esse problema vai além de enumerar uma lista de ações que ocorreram, fato impraticável sobre um grande volume de dados. Trata-se de criar representações gráficas que tornem observáveis estados e padrões por meio de "rápidas" inspeções visuais, nas quais as mudanças sejam evidenciadas.

Visando elaborar uma estratégia para representar o estado do ambiente, recorreuse às técnicas desenvolvidas por Lam (2005) nos softwares Seascape e Volcano para a visualização das interações na usenet. Suas pesquisas investigam o uso do movimento para representar informações de interações sociais. Assim, ele foca-se no uso de laços de animação periódicos para evidenciar a dinâmica dos dados.

Existem várias formas de utilizar o movimento como técnica de visualização de dados. Normalmente, ele é utilizado associado ao tempo histórico e linear. Em Seascape $e$ Volcano, o movimento não representa qualidades temporais das informações, mas sim concretiza o ritmo e atividade naquilo que o autor denomina timeless movement movimento sem início nem fim.

Como estudo de caso, foi desenvolvido um applet Java, denominado ProjectPulse, que implementa as técnicas desenvolvidas no Seascape para criar uma "paisagem" com as informações dos projetos de um ambiente criado no AMADIS.

\footnotetext{
4 Traduzido do inglês, information landscapes é um termo cunhado por Cooper (1994) que define um "ambiente digital onde a informação está geograficamente posicionada em um espaço virtual".

$6 \longrightarrow$ V. 4 No 1, Julho, 2006
} 


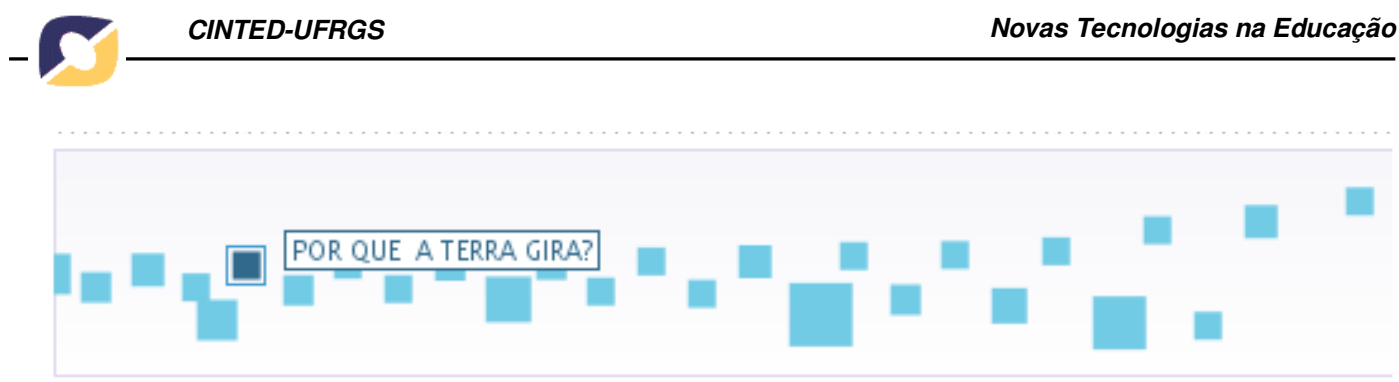

Figura 2: Applet ProjectPulse exibindo o nome de um projeto.

Como pode ser observado na Figura 2, no ProjectPulse, existem vários quadrados no espaço da tela que movem-se constantemente, seguindo uma trilha definida por uma função senoidal. Cada uma dessas figuras é a representação de um projeto cadastrado no AMADIS, cujos atributos de movimento são definidos pelas seguintes regras: (a) o tamanho do quadrado é proporcional ao número de usuários do projeto, (b) a amplitude do movimento é definida com base na quantidade de interações que o projeto teve desde sua criação e (c) a velocidade do movimento é determinada pelo número de interações que o projeto teve nos últimos 7 dias.

Depois de se apropriar dessas regras, o usuário consegue, por meio de uma rápida inspeção visual, observar vários padrões de comportamento nos diferentes projetos. Por exemplo, os projeto que estão mais ativos se movem mais rápido do que os demais. Já os projetos que estiveram muito ativos no passado, mas hoje estão "adormecidos", movem-se com uma grande amplitude e de forma lenta. Já os projetos que foram criados recentemente e ainda não tiveram interação ou produção têm uma trajetória praticamente retilínea.

Por meio dessa ferramenta, o usuário consegue observar mudanças nos padrões de movimentação das figuras geométricas ao longo do tempo. Torna-se mais fácil inferir o que está ocorrendo na totalidade do ambiente, sem a necessidade de inspecionar cada um dos projetos individualmente. Conforme aumenta a velocidade dos quadrados, mais ativo encontra-se o ambiente.

Nas próximas etapas para esta linha de investigação do AMADIS, deseja-se explorar novas métricas para definir a diferença entre um projeto ativo e um projeto inativo no ambiente. Até então, esse valor foi definido por uma simples contagem, devido à facilidade de implementação. Atualmente, encontra-se em desenvolvimento uma visualização para os usuários da plataforma, por meio da utilização das técnicas definidas para o sistema Volcano, acima citado.

Outro experimento realizado no AMADIS, envolvendo o uso de técnicas de visualização de informações, foi realizado utilizando a ferramenta de chat. Desejava-se criar uma visualização das sessões de chat encerradas, a partir das seguintes características: (a) visualizar todas as contribuições do sujeito sem perder a referência com o contexto da sala de chat, (b) agregar informações sobre o histórico de participações do usuário ao longo do tempo, (c) representar as diversas contribuições do usuário em forma gráfica para facilitar o reconhecimento de padrões e (d) possibilitar o mapeamento visual das conversações paralelas. 


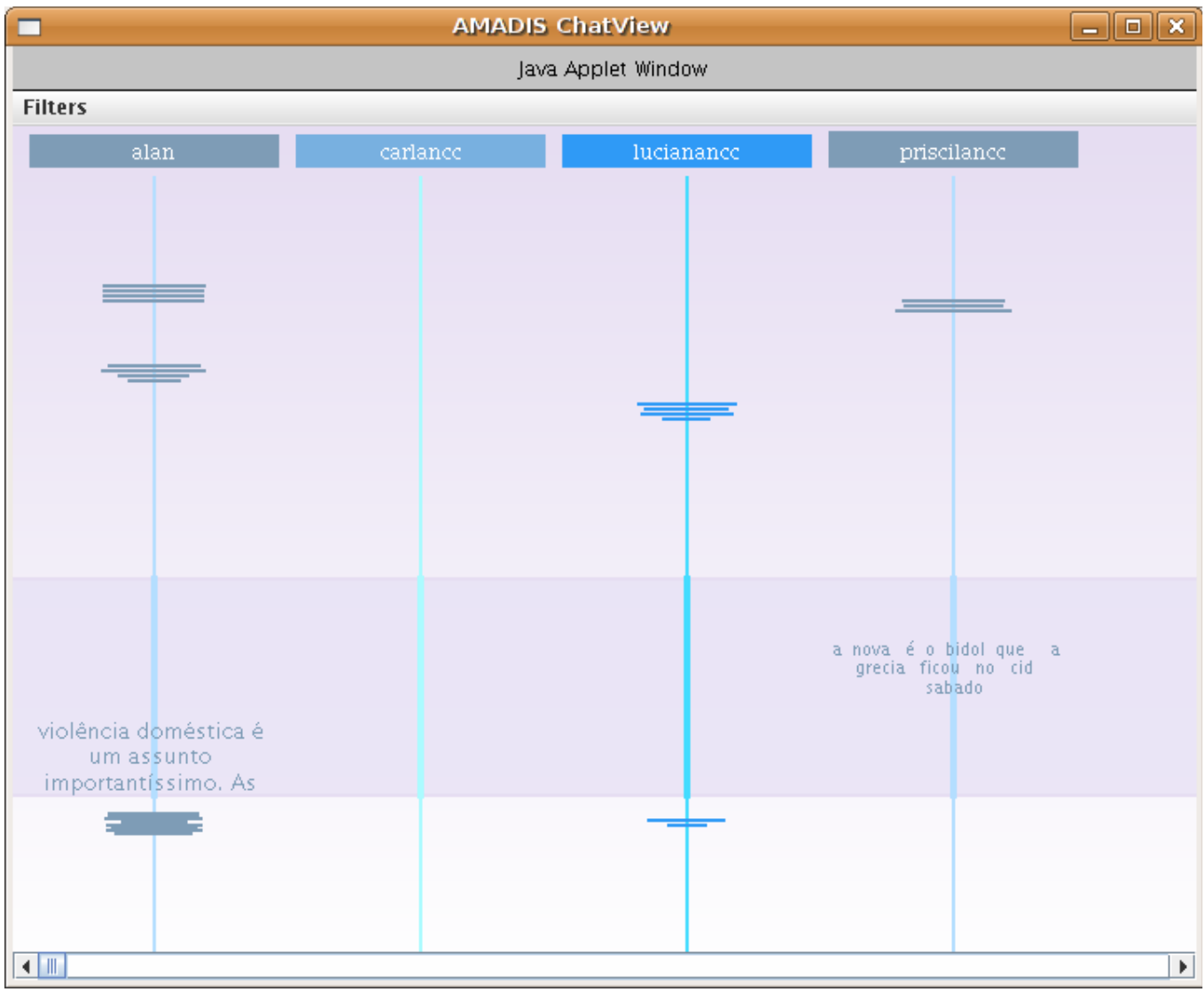

Figura 3: Tela do ChatView exibindo o log de um chat.

Tais parâmetros foram estabelecidos para se tentar, entre outros objetivos, amenizar os problemas, apontados por Garcia e Jacobs(1999), de impossibilidade da reconstrução do contexto de um chat apenas pela análise de seus logs. Essas duas autoras, embasadas na análise da conversação, apontam que, no chat, o usuário não tem um controle exato do posicionamento de suas mensagens, na medida em que tal posição é parcialmente controlada pelo próprio software, que organiza a sequiência das mensagens segundo a ordem de chegada e não de produção(emissão). Assim, elas afirmam que no chat a produção e o envio das mensagens são dissociados, ao contrário da conversação verbal. Devido a isso, elas apontam o chat como uma ferramenta quasesíncrona, pois demanda a participação simultânea, embora não garanta o ordenamento das mensagens.

Esse aspecto quase-síncrono do chat cria um efeito colateral chamado responsividade fantasma, que se caracteriza pela não seqüencialidade dos turnos de uma conversação. Esse fenômeno pode ser facilmente observado quando duas pessoas engajadas em um chat possuem várias linhas de conversas simultâneas. Em uma sala com várias pessoas, tal característica usualmente gera confusão e impossibilita a compreensão dos logs.

Como um primeiro experimento, tomou-se como base o trabalho de Viegas e Donath (1999) sobre o desenvolvimento de um cliente de chat gráfico, no qual foi utilizada a técnica de table lens ${ }^{5}$, para a visualização de $\log s$. Na implementação a seguir descrita, utilizou-se a mesma abordagem agregando-se a manipulação tipográfica

5 cf. Rao e Card(1994). 
das fontes para representar qualidades dos usuários, como descrito por Boyd (2002).

A visualização dos logs no AMADIS pode ser realizada utilizando-se a ferramenta ChatView. Como pode ser observado na Figura 3, o chat é exibido como uma tabela na qual cada usuário é representado por uma coluna e o tempo, pelas linhas. As mensagens de cada usuário são exibidas como células de sua respectiva coluna. Assim, observando apenas uma coluna, é possível ver toda a produção de um mesmo sujeito ao longo do chat. As mensagens, entretanto, são exibidas em tamanho reduzido, de forma que uma porção maior do diálogo seja visível simultaneamente. Para conseguir visualizar o conteúdo das mensagens, deve-se movimentar uma barra que funciona como uma lente, realizando um zoom sobre algumas linhas da tabela.

Outra característica dessa ferramenta é a fonte utilizada para renderizar as mensagens dos usuários. Tentou-se utilizar as fontes para representar propriedades sociais das interações de um usuário durante todos os seus chats. Como estudo de caso, foram eleitas duas propriedades quantitativas devido à facilidade de implementação, são elas: a quantidade de mensagens enviadas pelo usuário em todos os chats que participou e o tamanho médio de suas mensagens. Na Figura 3, pode-se observar a relação do tamanho da fonte com a quantidade de mensagens enviadas.

Tais propriedades da renderização das fontes tentam "contar" um pouco da história do usuário na interação com o ambiente. Apesar das métricas até então utilizadas serem estritamente quantitativas, deseja-se explorar novas possibilidades em próximas versões.

Outro desenvolvimento futuro é traçar linhas entre as mensagens que façam parte de uma mesma conversação, tentando traçar a sequiência ou a rede das trocas de turnos. Para tanto, pretende-se utilizar elementos teóricos da análise da conversação como os pares conversacionais. Considerando que o sistema possivelmente cometerá equívoco, pretende-se permitir que o usuário manipule tais ligações.

\section{Considerações Finais}

O Projeto AMADIS ainda é uma pesquisa em desenvolvimento. As reflexões aqui relatadas têm como objetivo divulgar os resultados preliminares dos experimentos realizados nessa plataforma. Acredita-se que as iniciativas aqui descritas tragam contribuições para a comunidade acadêmica no que se refere ao desenvolvimento de plataformas virtuais de aprendizagem.

Também se desejam destacar, com o presente texto, as dificuldades envolvidas na construção de uma proposta de plataforma que se afaste de um modelo sustentado somente por cursos e conteúdos programáticos. A tendência em repetir no virtual os modelos cristalizados da escola tradicional é forte. Rompê-los exige constante reflexão sobre a coerência da teoria com a prática realizada.

\section{Referências}

BOYD, Danah e LEE, Hyun-Yeul e RAMAGE, Daniel e DONATH, Judith S. Developing Legible Visualisations for Online Social Spaces.In: HICSS, 2002, Hawaii. Anais.

COOPER, Muriel, et.al. "Information Landscapes". MIT Technical Note. 2004. 
FAGUNDES, Léa., MAÇADA, D., Sato, L; Aprendizes do Futuro, as Inovações Começaram. MEC, 1999.

FAGUNDES, Léa; NEVADO, Rosane; MENEZES, Crediné; MONTEIRO, Valéria. Ferramenta de Autoria e Interação para apoio ao desenvolvimento de Projetos de Aprendizagem. CINTED-UFRGS, 2005.

GARCIA, Angela e Jacobs, J.B. The Eyes of the Beholder: undestanding the turn-taking system in quasi-synchronous Computer-Mediated Communication. In: Research on Language and Social Interaction, 32, nº 4, p. 337-367, 1999.

GERSHON, N. e EICK, S. G. Information Visualization. In: IEEE Computer Graphics and Applications, 17, $\mathrm{n}^{\circ}$ 4, p. 29-31, 1997.

LAM, Francis e DONATH, Judith. Seascape and Volcano: Visualizing Online Discussions Using Timeless Motion. In: CHI '05: CHI '05 extended abstracts on Human factors in computing systems, 2005, Portland, USA. Anais. Nova York: ACM Press p. 1585-1588.

MARCUSCHI, Luiz Antonio. Analise da Conversação. São Paulo: ATICA, 2003.

NEVADO, Rosane; BASSO, Marcus V.; BITTENCOURT, Juliano de V. AMADIS: Ambiente de Aprendizagem a Distância para Formação Continuada de Professores. In: Revista Informática na Educação - Teoria e Prática, vol. 4, $\mathrm{n}^{\circ}$ 2, PGIEUFRGS, 2001.

NEVADO, Rosane, MAGDALENA, Beatriz e COSTA, Íris. Formação de Professores Multiplicadores: nte2@ projetos.cooperativos.ufrgs.br. In: Revista Informática na Educação - Teoria e Prática, vol. 2 nº 2, PGIE-UFRGS, 1999.

RAO, Ramana e CARD, Stuart K. The table lens: merging graphical and symbolic representations in an interactive focus + context visualization for tabular information. In: CHI '94: Proceedings of the SIGCHI conference on Human factors in computing systems, 1994, Boston, USA. Anais. Nova York: ACM Press, p. 318-322.

VIEGAS, Fernanda B e DONATH, Judith S. Chat Circles. In: Proceedings of the SIGCHI conference on Human factors in computing systems, 1999, Pittsburgh, USA. Anais. Nova York: ACM Press. p. 9-16. 